\title{
LIMIT CYCLES BIFURCATING FROM A ZERO-HOPF SINGULARITY IN ARBITRARY DIMENSION
}

\author{
LUIS BARREIRA ${ }^{1}$, JAUME LLIBRE $^{2}$ AND CLAUDIA VALLS ${ }^{1}$
}

\begin{abstract}
We study the limit cycles which can bifurcate from a zeroHopf singularity of a $C^{m+1}$ differential system in $\mathbb{R}^{n}$, i.e. from a singularity with eigenvalues $\pm b i$ and $n-2$ zeros for $n \geq 3$. If this singularity is at the origin of coordinates and the Taylor expansion of the differential system at the origin without taking into account the linear terms starts with terms of order $m$, from the origin it can bifurcate $s$ limit cycles with $s \in\left\{0,1, \ldots, 2^{n-3}\right\}$ if $m=2$ (see [7]), with $s \in\left\{0,1, \ldots, 3^{n-2}\right\}$ if $m=3$, with $s \leq 6^{n-2}$ if $m=4$, and with $s \leq 4 \cdot 5^{n-2}$ if $m=5$. Moreover, $s \in\{0,1,2\}$ if $m=4$ and $n=3$, and $s \in\{0,1,2,3,4,5\}$ if $m=5$ and $n=3$. Note that the maximum number of limit cycles bifurcating from this zero-Hopf singularity grows up exponentially with the dimension for $m=2,3$.
\end{abstract}

\section{Introduction AND StATEMENT OF the MAIN RESUlts}

Zero-Hopf singularity is an isolated equilibrium point of an $n$-dimensional autonomous differential systems with $n \geq 3$, such that its linear part has $n-2$ zeros eigenvalues and a pair of purely imaginary eigenvalues. The unfolding has complex dynamics in the small neighborhood of this isolated equilibrium, see for instance Guckenheimer and Holmes [3, 4], Scheurle and Marsden [11], Kuznetsov in [5] and references therein. It has been shown that some complicated invariant sets of the unfolding could be bifurcated from the isolated zero-Hopf equilibrium under some conditions. Hence, zero-Hopf bifurcation could imply a local birth of "chaos" (cf. $[2,11])$.

We study the zero-Hopf bifurcation of $C^{m+1}$ differential systems in $\mathbb{R}^{n}$ with $n \geq 3$ and $m=2,3,4,5$ by using the first-order averaging theory. Thus we assume that these systems have a singularity at the origin, whose linear part has eigenvalues $\varepsilon a \pm b i$ and $\varepsilon c_{k}$ for $k=3, \ldots, n$, where $\varepsilon$ is a small

2010 Mathematics Subject Classification. Primary 37J35, 37K10.

Key words and phrases. ?? 
parameter. Such systems can be written in the form

$$
\begin{aligned}
\dot{x} & =\varepsilon a x-b y+\sum_{i_{1}+\ldots+i_{n}=m} a_{i_{1} \cdots i_{n}} x^{i_{1}} y^{i_{2}} z_{3}^{i_{3}} \cdots z_{n}^{i_{n}}+\mathcal{A}, \\
\dot{y} & =b x+\varepsilon a y+\sum_{i_{1}+\ldots+i_{n}=m} b_{i_{1} \cdots i_{n}} x^{i_{1}} y^{i_{2}} z_{3}^{i_{3}} \cdots z_{n}^{i_{n}}+\mathcal{B}, \\
\dot{z}_{k} & =\varepsilon c_{k} z_{k}+\sum_{i_{1}+\ldots+i_{n}=m} c_{i_{1} \cdots i_{n}}^{(k)} x^{i_{1}} y^{i_{2}} z_{3}^{i_{3}} \cdots z_{n}^{i_{n}}+\mathcal{C}_{k}, \quad k=3, \ldots, n,
\end{aligned}
$$

where $a_{i_{1}, \cdots, i_{n}}, b_{i_{1}, \cdots, i_{n}}, c_{i_{1} \cdots i_{n}}^{(k)}, a, b$ and $c_{k}$ are real parameters, $a b \neq 0$ and $\mathcal{A}, \mathcal{B}$ and $\mathcal{C}_{k}$ are continuous functions given by the Lagrange expressions of the error in the expansion of order $m+1$ of the system in Taylor series.

Our main result is on the number of limit cycles which can bifurcate from the origin in a zero-Hopf bifurcation by the first-order averaging method. The study of limit cycles and averaging theory has a long history, see for instance $[1,9,10,13]$ and the references therein. As far as we know our main result (see Theorem 2 for $m=3$ ) confirms that the number of bifurcated limit cycles in a zero-Hopf bifurcation can grow exponentially with the dimension of the system. In the particular case of $m=2$ and also for the zero-Hopf bifurcation a similar result was proved in [7]. More precisely they proved the following result.

Theorem 1. If $m=2$ there exist $C^{3}$ differential systems (1) for which $\ell$ limit cycles, with $\ell \in\left\{0,1, \ldots, 2^{n-3}\right\}$, bifurcate from the origin at $\varepsilon=0$. That is, for $\varepsilon>0$ sufficiently small there are systems having exactly $\ell$ limit cycles in a neighborhood of the origin and these limit cycles tend to the origin when $\varepsilon \searrow 0$.

The first main result of the paper is the following.

Theorem 2. If $m=3$ there exist $C^{4}$ differential systems (1) for which $\ell$ limit cycles, with $\ell \in\left\{0,1, \ldots, 3^{n-2}\right\}$, bifurcate from the origin at $\varepsilon=0$. That is, for $\varepsilon>0$ sufficiently small there are systems having exactly $\ell$ limit cycles in a neighborhood of the origin and these limit cycles tend to the origin when $\varepsilon \searrow 0$.

Theorem 2 is proved in section 3. The particular case of Theorem 2 for $n=3$ was proved in [6] where the authors provide also the kind of stability of the limit cycles.

Now we consider the case $m=4$ and $m=5$.

Theorem 3. If $m=4$ for $\varepsilon>0$ sufficiently small the $C^{5}$ differential systems (1) can have at most $6^{n-2}$ limit cycles in a neighborhood of the origin, and these limit cycles tend to the origin when $\varepsilon \searrow 0$. When $n=3$ the maximum number of limit cycles which can bifurcate from the origin is 2 and this bound is reached. 
The proof of Theorem 3 is given in section 4 .

Theorem 4. If $m=5$ for $\varepsilon>0$ sufficiently small the $C^{6}$ differential systems (1) can have at most $4 \cdot 5^{n-2}$ limit cycles in a neighborhood of the origin, and these limit cycles tend to the origin when $\varepsilon \searrow 0$. When $n=3$ the maximum number of limit cycles which can bifurcate from the origin is 5 and this bound is reached.

The proof of Theorem 4 is given in section 5 .

\section{The FIRST-ORDER AVERAgING METHOD FOR PERIODIC ORBITS}

The aim of this section is to present the first-order averaging method as it was obtained in [1] (see also [10] for a differential version and the general averaging theory). Basically this method gives a quantitative relation between the solutions of some non-autonomous periodic differential system and the singularities of its averaged differential system. Differentiability of the vector field is not needed. The specific conditions for the existence of a simple isolated zero of the averaged function are given in terms of the Brower degree. In fact the Brouwer degree theory is the key point in the proof of this theorem.

Theorem 5. Consider the following differential system

$$
\dot{x}(t)=\varepsilon f(t, x)+\varepsilon^{2} R(t, x, \varepsilon)
$$

where $f: \mathbb{R} \times D, R: \mathbb{R} \times D \times\left(-\varepsilon_{f}, \varepsilon_{f}\right) \rightarrow \mathbb{R}^{n}$ are continuous functions, $T$ periodic in the first variable, and $D$ is a bounded open subset of $\mathbb{R}^{n}$. Define $f^{0}: D \rightarrow \mathbb{R}^{n}$ as

$$
f^{0}(z)=\frac{1}{T} \int_{0}^{T} f(s, z) d s
$$

and assume that

(i) $f$ and $R$ are locally Lipschitz with respect to $x$;

(ii) for $v \in D$ with $f^{0}(v)=0$, there exists a neighborhood $V$ of $v$ such that $f^{0}(z) \neq 0$ for all $z \in \bar{V} \backslash\{v\}$ and $d_{B}\left(f^{0}, V, v\right) \neq 0$ (where $d_{B}\left(f^{0}, V, v\right)$ denotes the Brower degree of $f^{0}$ in a neighborhood $V$ of $v)$.

Then for $|\varepsilon|>0$ sufficiently small there exists an isolated T-periodic solution $x(t, \varepsilon)$ of system (2) such that $x(0, \varepsilon) \rightarrow v$ when $\varepsilon \rightarrow 0$. Moreover if the function $f$ in (2) is $C^{2}$ and the function $R$ is $C^{1}$ in a neighborhood of a simple zero $v$ of $f^{0}$ the stability or instability of the limit cycle $x(t, \varepsilon)$, is given by the stability or instability of the singularity $v$ of the averaged system $\dot{z}=\varepsilon f^{0}(z)$. If all the eigenvalues of the Jacobian matrix of $f^{0}$ at $v$ have negative real part, then the limit cycle $x(t, \varepsilon)$ is asymptotically stable. If some of these eigenvalues has positive real part then the limit cycle $x(t, \varepsilon)$ is unstable. 
We recall that if $f^{0}$ is of class $C^{1}$ and the determinant of the Jacobian matrix at the zero $v$ is nonzero, then $d_{B}\left(f^{0}, V, v\right) \neq 0$, and the zeroes are called simple zeroes of $f^{0}$, see [8].

\section{Proof of Theorem 2}

Doing the cylindrical change of variables

$$
x=r \cos \theta, \quad y=r \sin \theta, \quad z_{i}=z_{i}, \quad i=3, \ldots, n
$$

in the region $r>0$, system (1) becomes

$(5)$

$$
\begin{aligned}
\dot{r} & =\varepsilon a r+\sum\left(a_{i_{1} \cdots i_{n}} \cos \theta+b_{i_{1} \cdots i_{n}} \sin \theta\right)(r \cos \theta)^{i_{1}}(r \sin \theta)^{i_{2}} z_{3}^{i_{3}} \cdots z_{n}^{i_{n}}+O_{4}, \\
\dot{\theta} & =\frac{1}{r}\left(b r+\sum\left(b_{i_{1} \cdots i_{n}} \cos \theta-a_{i_{1} \cdots i_{n}} \sin \theta\right)(r \cos \theta)^{i_{1}}(r \sin \theta)^{i_{2}} z_{3}^{i_{3}} \cdots z_{n}^{i_{n}}+O_{4},\right. \\
\dot{z}_{k} & =\varepsilon c_{k} z_{k}+\sum c_{i_{1} \cdots i_{n}}^{(k)}(r \cos \theta)^{i_{1}}(r \sin \theta)^{i_{2}} z_{3}^{i_{3}} \cdots z_{n}^{i_{n}}+O_{4}, \quad k=3, \ldots, n,
\end{aligned}
$$

where $O_{4}=O_{4}\left(r, z_{3}, \ldots, z_{n}\right)$ and the sums goes over $i_{1}+\ldots+i_{n}=3$. As usual $\mathbb{Z}_{+}$denotes the set of all non-negative integers. Taking $a_{00 e_{i j}}=$ $b_{00 e_{i j}}=0$ where $e_{i j} \in \mathbb{Z}_{+}^{n-2}$ has the sum of the entries equal to 3 , it is easy to show that in a suitable neighborhood of $\left(r, z_{3}, \ldots, z_{n}\right)=(0,0, \ldots, 0)$ with $r>0$ we have $\dot{\theta} \neq 0$ because $b \neq 0$. Then choosing $\theta$ as the new independent variable, system $(5)$ in a neighborhood of $\left(r, z_{3}, \ldots, z_{n}\right)=(0,0, \ldots, 0)$ with $r>0$ becomes

(6)

$$
\begin{aligned}
\frac{d r}{d \theta} & =\frac{r\left(\varepsilon a r+\sum\left(a_{i_{1} \cdots i_{n}} \cos \theta+b_{i_{1} \cdots i_{n}} \sin \theta\right)(r \cos \theta)^{i_{1}}(r \sin \theta)^{i_{2}} z_{3}^{i_{3}} \cdots z_{n}^{i_{n}}+O_{4}\right.}{b r+\sum\left(b_{i_{1} \cdots i_{n}} \cos \theta-a_{i_{1} \cdots i_{n}} \sin \theta\right)(r \cos \theta)^{i_{1}}(r \sin \theta)^{i_{2}} z_{3}^{i_{3}} \cdots z_{n}^{i_{n}}+O_{4}}, \\
\frac{d z_{k}}{d \theta} & =\frac{r\left(\varepsilon c_{k} z_{k}+\sum c_{i_{1} \cdots i_{n}}(r) \cos \theta\right)^{i_{1}}(r \sin \theta)^{i_{2}} z_{3}^{i_{3}} \cdots z_{n}^{i_{n}}+O_{4}}{b r+\sum\left(b_{i_{1} \cdots i_{n}} \cos \theta-a_{i_{1} \cdots i_{n}} \sin \theta\right)(r \cos \theta)^{i_{1}}(r \sin \theta)^{i_{2}} z_{3}^{i_{3}} \cdots z_{n}^{i_{n}}+O_{4}},
\end{aligned}
$$

for $k=3, \ldots, n$, where the sum runs over $i_{1}+\cdots+i_{n}=3$. Note that this system is periodic or period $2 \pi$ in $\theta$.

To apply the averaging theory in the proof of Theorem 2 we rescale the variables, setting

$$
\left(r, z_{3}, \ldots, z_{n}\right)=\left(\varepsilon^{1 / 2} \rho, \varepsilon^{1 / 2} \eta_{3}, \ldots, \varepsilon^{1 / 2} \eta_{n}\right) .
$$

Then system (1) becomes

$$
\begin{aligned}
\frac{d \rho}{d \theta} & =\varepsilon f_{1}\left(\theta, \rho, \eta_{3}, \ldots, \eta_{n}\right)+\varepsilon^{2} g_{1}\left(\theta, \rho, \eta_{3}, \ldots, \eta_{n}\right), \\
\frac{d \eta_{k}}{d \theta} & =\varepsilon f_{k}\left(\theta, \rho, \eta_{3}, \ldots, \eta_{n}\right)+\varepsilon^{2} g_{k}\left(\theta, \rho, \eta_{3}, \ldots, \eta_{n}\right),
\end{aligned}
$$


for $k=3, \ldots, n$ where

$$
\begin{aligned}
& f_{1}=\frac{1}{b}\left(a \rho+\sum_{i_{1}+\cdots+i_{n}=3}\left(a_{i_{1} \cdots i_{n}} \cos \theta+b_{i_{1} \cdots i_{n}} \sin \theta\right)(\rho \cos \theta)^{i_{1}}(\rho \sin \theta)^{i_{2}} z_{3}^{i_{3}} \cdots z_{n}^{i_{n}}\right), \\
& f_{k}=\frac{1}{b}\left(c \eta_{k}+\sum_{i_{1}+\cdots+i_{n}=3} c_{i_{1} \cdots i_{n}}^{(k)}(\rho \cos \theta)^{i_{1}}(\rho \sin \theta)^{i_{2}} z_{3}^{i_{3}} \cdots z_{n}^{i_{n}}\right) .
\end{aligned}
$$

Then system (8) has the normal form (2) for applying the averaging theory with $x=\left(\rho, \eta_{3}, \ldots, \rho_{n}\right), t=\theta, T=2 \pi$, and

(9)

$$
f\left(\theta, \rho, \eta_{3}, \ldots, \eta_{n}\right)=\left(f_{1}\left(\theta, \rho, \eta_{3}, \ldots, \eta_{n}\right), f_{3}\left(\theta, \rho, \eta_{3}, \ldots, \eta_{n}\right), \ldots, f_{n}\left(\theta, \rho, \eta_{3}, \ldots, \eta_{n}\right)\right) .
$$

The averaged system of system (8) is

$$
\dot{y}=\varepsilon f^{0}(y), \quad y=\left(\rho, \eta_{3}, \ldots, \eta_{n}\right) \in \Omega,
$$

where $\Omega$ is suitable neighborhood of the origin $\left(\rho, \eta_{3}, \ldots, \rho_{n}\right)=(0,0, \ldots, 0)$ with $\rho>0$ and

$$
f^{0}(y)=\left(f_{1}^{0}(y), f_{3}^{0}(y), \ldots, f_{n}^{0}(y)\right)
$$

with

$$
f_{i}^{0}(y)=\frac{1}{2 \pi} \int_{0}^{2 \pi} f_{i}\left(\theta, \rho_{3}, \ldots, \rho_{n}\right) d \theta, \quad i=1,3, \ldots, n .
$$

After some calculations we obtain

$$
\begin{aligned}
f_{1}^{0}= & \frac{1}{8 b} \rho\left(8 a+\left(a_{12 \mathbf{0}_{n-2}}+b_{21 \mathbf{0}_{n-2}}+3\left(a_{30 \mathbf{0}_{n-2}}+b_{03 \mathbf{0}_{n-2}}\right)\right) \rho^{2}\right. \\
& \left.+4 \sum_{3 \leq i \leq j \leq n}^{n}\left(a_{10 e_{i j}}+b_{01 e_{i j}}\right) \eta_{i} \eta_{j}\right), \\
f_{k}^{0}= & \frac{1}{2 b}\left(2 c_{k} \eta_{k}+\sum_{j=3}^{n}\left(c_{20 e_{j}}^{(k)}+c_{02 e_{j}}^{(k)}\right) \rho^{2} \eta_{j}+2 \sum_{3 \leq i \leq j \leq l \leq n} c_{00 e_{i j l}}^{(k)} \eta_{i} \eta_{j} \eta_{l}\right),
\end{aligned}
$$

for $k=3, \ldots, n$, where $e_{j} \in \mathbb{Z}_{+}^{n-2}$ is the unit vector with the $j$-entry equal to $1, e_{i j} \in \mathbb{Z}_{+}^{n-2}$ has the sum of the $i$-th and $j$-th entries equal to 2 and the other equal to 0 (note that eventually $i$ can be equal to $j$ ), and $e_{i j l} \in \mathbb{Z}_{+}^{n-2}$ has the sum of the $i$-th, $j$-th and $l$-th entries equal to 3 and the other entries equal to zero (again eventually some $i, j$ and $l$ can coincide).

Now we apply Theorem 5 for obtaining limit cycles of system (8). After the rescaling (7), these limits will become infinitesimal limit cycles for system (6), which will tend to origin when $\varepsilon \searrow 0$, consequently they will be limit cycles bifurcating from the zero-Hopf bifurcation of system (1) at the origin.

Using Theorem 5 for studying the limit cycles of system (8) we only need to compute the simple singularities of system (10). Since the transformation from the cartesian coordinates $\left(x, y, z_{3}, \ldots, z_{n}\right)$ to the cylindrical ones $\left(r, \theta, z_{3}, \ldots, z_{n}\right)$ is not a diffeomorphism at $r=0$, we deal with the zeros of 
the averaged function $f^{0}$ of (12) having the coordinate $\rho>0$. So we need to compute the zeroes of the algebraic equations

$$
\begin{aligned}
& 8 a+\left(a_{12 \mathbf{0}_{n-2}}+b_{21 \mathbf{0}_{n-2}}+3\left(a_{30 \mathbf{0}_{n-2}}+b_{03 \mathbf{0}_{n-2}}\right)\right) \rho^{2} \\
& +4 \sum_{3 \leq i \leq j \leq n}^{n}\left(a_{10 e_{i j}}+b_{01 e_{i j}}\right) \eta_{i} \eta_{j}=0, \\
& 2 c_{k} \eta_{k}+\sum_{j=3}^{n}\left(c_{20 e_{j}}^{(k)}+c_{02 e_{j}}^{(k)}\right) \rho^{2} \eta_{j}+2 \sum_{3 \leq i \leq j \leq l \leq n} c_{00 e_{i j l}}^{(k)} \eta_{i} \eta_{j} \eta_{l}=0,
\end{aligned}
$$

for $k=3, \ldots, n$. Since the coefficients of this system are independent and arbitrary, we can simplify the notation by writing the system as

$$
\begin{aligned}
& a+a_{1} \rho^{2}+\sum_{3 \leq i \leq j \leq n}^{n} a_{i j} \eta_{i} \eta_{j}=0, \\
& c_{k} \eta_{k}+\sum_{j=3}^{n} c_{j}^{(k)} \rho^{2} \eta_{j}+\sum_{3 \leq i \leq j \leq l \leq n} c_{i j l}^{(k)} \eta_{i} \eta_{j} \eta_{l}=0,
\end{aligned}
$$

for $k=3, \ldots, n$, where $a_{1}, a_{i j}, c_{j}^{(k)}$ and $c_{i j l}^{(k)}$ are arbitrary constants. Denote by $\mathcal{C}$ the set of algebraic systems of form (14).

We claim that there is a system in $\mathcal{C}$ which has exactly $3^{n-2}$ simple zeros. The claim can be verified by the example

$$
\begin{gathered}
a+a_{1} \rho^{2}=0, \\
c_{k} \eta_{k}+\sum_{j=3}^{k} c_{j}^{(k)} \rho^{2} \eta_{j}+\sum_{3 \leq i \leq j \leq l \leq k} c_{i j l}^{(k)} \eta_{i} \eta_{j} \eta_{l}=0, \quad k=3, \ldots, n
\end{gathered}
$$

with all the coefficients nonzero. Equation (15) is a linear equation in $\rho^{2}$, and equation (16) is a cubic algebraic equation in the $\eta_{j}$ 's. Substituting the unique positive solution $\rho_{0}$ of (15) into (16) with $k=3$, then this last equation has exactly three different real solutions $\eta_{30}, \eta_{31}$ and $\eta_{32}$ choosing conveniently the coefficients $c_{3}^{(3)}$ and $c_{333}^{(3)}$. Introducing one of the four solutions $\left(\rho_{0}, \eta_{3 i}\right), i=0,1,2$, into (16) with $k=4$, choosing conveniently the values of the coefficients of (16) with $k=4$ we get three different solutions $\eta_{40}^{i}, \eta_{41}^{i}$ and $\eta_{42}^{i}$ of $\eta_{4}$. Moreover playing with the coefficients of the equations, the nine solutions $\left(\eta_{3 i}, \eta_{40}^{i}, \eta_{41}^{i}, \eta_{42}^{i}\right)$ for $i=0,1,2$ are distinct. Repeating this process we can prove that for suitable choice of the coefficients of equations (15) and (16), these equations have $3^{n-2}$ different zeroes. Since $3^{n-2}$ solutions of (15) and (16) is the maximum that the equations (14) can have by Bezout Theorem (see [12]), it follows that every solution is simple, and consequently the determinant of the Jacobi of the system evaluated at it is non-zero. This proves the claim. 
Using the same arguments which allow to prove the claim, we also can prove that we can choose the coefficients of the previous system in order that it has $s$ simple real solutions with $s \in\left\{0,1, \ldots, 3^{n-2}\right\}$.

Taking the averaged system (10) with $f^{0}$ having the convenient coefficients as in (15)-(16), the averaged system (10) has exactly $s \in\left\{0,1, \ldots, 3^{n-2}\right\}$ singularities with $\rho>0$. Moreover the determinants of the Jacobian matrix $\partial f^{0} / \partial y$ at these singularities do not vanish, because all the singularities are simple. By Theorem 5 we get that there are systems (1) which have $s \in\left\{0,1, \ldots, 3^{n-2}\right\}$ limit cycles bifurcating from the origin of coordinates. This proves Theorem 2 .

\section{Proof of Theorem 3}

Doing the cylindrical change of variables in (4) in the region $r>0$, the system (1) becomes system (5) where now the sum runs over $i_{1}+\cdots+$ $i_{n}=4$. Taking $a_{00 e_{i j}}=b_{00 e_{i j}}=0$ where $e_{i j} \in \mathbb{Z}_{+}^{n-2}$ has the sum of the entries equal to 4 , it is easy to show that in a suitable neighborhood of $\left(r, z_{3}, \ldots, z_{n}\right)=(0,0, \ldots, 0)$ we have that $\dot{\theta} \neq 0$. Then choosing $\theta$ as the new independent variable system $(5)$ in a neighborhood of $\left(r, z_{3}, \ldots, z_{n}\right)=$ $(0,0, \ldots, 0)$ becomes system $(6)$ where again the sum runs over $i_{1}+\cdots+i_{n}=$ 4. Note that this system is periodic or period $2 \pi$ in $\theta$.

To apply the averaging theory in the proof of Theorem 3 we rescale the variables, setting

$$
\left(r, z_{3}, \ldots, z_{n}\right)=\left(\varepsilon^{1 / 3} \rho, \varepsilon^{1 / 3} \eta_{3}, \ldots, \varepsilon^{1 / 3} \eta_{n}\right)
$$

Then system (6) becomes system (8) with

$$
\begin{aligned}
& f_{1}=\frac{1}{b}\left(a \rho+\sum_{i_{1}+\cdots+i_{n}=4}\left(a_{i_{1} \cdots i_{n}} \cos \theta+b_{i_{1} \cdots i_{n}} \sin \theta\right)(\rho \cos \theta)^{i_{1}}(\rho \sin \theta)^{i_{2}} z_{3}^{i_{3}} \cdots z_{n}^{i_{n}}\right), \\
& f_{k}=\frac{1}{b}\left(c \eta_{k}+\sum_{i_{1}+\cdots+i_{n}=4} c_{i_{1} \cdots i_{n}}^{(k)}(\rho \cos \theta)^{i_{1}}(\rho \sin \theta)^{i_{2}} z_{3}^{i_{3}} \cdots z_{n}^{i_{n}}\right) .
\end{aligned}
$$

Then system (8) has the normal form (2) of the averaging theory with $x=$ $\left(\rho, \eta_{3}, \ldots, \rho_{n}\right), t=\theta, T=2 \pi$, and $f$ given in (9). The averaged system (8) is system (10) where $\Omega$ is suitable neighborhood of the origin $\left(\rho, \eta_{3}, \ldots, \rho_{n}\right)=$ $(0,0, \ldots, 0)$ with $\rho>0$ and $f^{0}(y)$ given in (11). After some computations 
we obtain

$$
\begin{aligned}
f_{1}^{0}= & \frac{1}{8 b} \rho\left(8 a+\sum_{j=3}^{n}\left(a_{12 e_{j}}+b_{21 e_{j}}+3\left(a_{30 e_{j}}+b_{03 e_{j}}\right)\right) \rho^{2} \eta_{j}\right. \\
& \left.+4 \sum_{3 \leq i \leq j \leq l \leq n}^{n}\left(a_{10 e_{i j l}}+b_{01 e_{i j l}}\right) \eta_{i} \eta_{j} \eta_{l}\right), \\
f_{k}^{0}= & \frac{1}{8 b}\left(8 c_{k} \eta_{k}+3 \sum_{j=3}^{n}\left(c_{04 \mathbf{0}_{n-2}}^{(k)}+c_{22 \mathbf{0}_{n-2}}^{(k)}+c_{40 \mathbf{0}_{n-2}}^{(k)}\right) \rho^{4}\right. \\
& \left.+4 \sum_{3 \leq i \leq j \leq n}\left(c_{20 e_{i j}}^{(k)}+c_{02 e_{i j}}^{(k)}\right) \rho^{2} \eta_{i} \eta_{j}+8 \sum_{3 \leq i \leq j \leq l \leq u \leq n} c_{00 e_{i j l u}}^{(k)} \eta_{i} \eta_{j} \eta_{l} \eta_{u}\right)
\end{aligned}
$$

for $k=3, \ldots, n$, where $e_{i j l u} \in \mathbb{Z}_{+}^{n-2}$ has the sum of the $i$-th, $j$-th, $l$-th and $u$-th entries equals to 4 and the others equal to 0 , eventually these entries can coincide.

Now we apply Theorem 5 for obtaining the limit cycles of system (8) (with the sum running over $i_{1}+\cdots+i_{n}=4$ ). After the rescaling (7), these limits will become infinitesimal limit cycles for system (6), which will tend to origin when $\varepsilon \searrow 0$, consequently they will be limit cycles bifurcating from the origin of the zero-Hopf bifurcation of system (1).

Using Theorem 5 for studying the limit cycles of system (8) we only need to compute the simple zeros of system (10) (with the sum running over $\left.i_{1}+\cdots+i_{n}=4\right)$. So we need to compute the zeroes of the algebraic equations

$$
\begin{aligned}
& 8 a+\sum_{j=3}^{n}\left(a_{12 e_{j}}+b_{21 e_{j}}+3\left(a_{30 e_{j}}+b_{03 e_{j}}\right)\right) \rho^{2} \eta_{j} \\
& +4 \sum_{3 \leq i \leq j \leq l \leq n}^{n}\left(a_{10 e_{i j l}}+b_{01 e_{i j l}}\right) \eta_{i} \eta_{j} \eta_{l}=0 \\
& 8 c_{k} \eta_{k}+3 \sum_{j=3}^{n}\left(c_{04 \mathbf{0}_{n-2}}^{(k)}+c_{22 \mathbf{0}_{n-2}}^{(k)}+c_{40 \mathbf{0}_{n-2}}^{(k)}\right) \rho^{4}+ \\
& 4 \sum_{3 \leq i \leq j \leq n}\left(c_{20 e_{i j}}^{(k)}+c_{02 e_{i j}}^{(k)}\right) \rho^{2} \eta_{i} \eta_{j}+8 \sum_{3 \leq i \leq j \leq l \leq u \leq n} c_{00 e_{i j l u}}^{(k)} \eta_{i} \eta_{j} \eta_{l} \eta_{u}=0,
\end{aligned}
$$

for $k=3, \ldots, n$.

Isolating $\rho$ from the first equation in (17), taking into account that $\rho>0$, and substituting it in the other equations of (17), the numerator of these equations become polynomial equations of degree 6 . Then by Bezout Theorem, the maximum number of solution that system (17) can have is $6^{n-2}$. We do not know if the bound is reached because regardless the fact that the coefficients are independent, in the first equation of (17) it appears $\rho^{2} \eta_{j}$ 
instead of $\rho^{2}$ as in the first equation of (13), so we cannot apply the same arguments as in the proof of Theorem 2, and we cannot show that the bounds are reached. We now consider the particular case of $\mathbb{R}^{3}$. In this case we have

$$
\begin{aligned}
f_{1}^{0} & =\frac{\rho}{8 b}\left(8 a+\left(a_{121}+b_{211}+3\left(a_{301}+b_{031}\right)\right) \rho^{2} \eta_{3}+4\left(a_{103}+b_{013}\right) \eta_{3}^{3}\right) \\
f_{2}^{0} & =\frac{1}{8 b}\left(8 c_{3} \eta_{3}+\left(3 c_{040}^{(3)}+c_{220}^{(3)}+3 c_{400}^{(3)}\right) \rho^{4}+4\left(c_{022}^{(3)}+c_{202}^{(3)}\right) \rho^{2} \eta_{3}^{2}+8 c_{004}^{(3)} \eta_{3}^{4}\right) .
\end{aligned}
$$

We need to compute the zeroes of

$$
\begin{aligned}
& 8 a+\left(a_{121}+b_{211}+3\left(a_{301}+b_{031}\right)\right) \rho^{2} \eta_{3}+4\left(a_{103}+b_{013}\right) \eta_{3}^{3}=0, \\
& 8 c_{3} \eta_{3}+\left(3 c_{040}^{(3)}+c_{220}^{(3)}+3 c_{400}^{(3)}\right) \rho^{4}+4\left(c_{022}^{(3)}+c_{202}^{(3)}\right) \rho^{2} \eta_{3}^{2}+8 c_{004}^{(3)} \eta_{3}^{4}=0 .
\end{aligned}
$$

Setting $\rho^{2}=R$ from the first equation we get

$$
R=-\frac{4\left(2 a+\left(a_{103}+b_{013}\right) \eta_{3}^{3}\right)}{\eta_{3}\left(a_{121}+3 a_{301}+3 b_{031}+b_{211}\right)} .
$$

Substituting $\rho^{2}$ into the second equation and taking the numerator we get a polynomial of degree two in the variable $\eta_{3}^{3}$. It has two solutions. Note that there is only one real solution for the cubic equation $x^{3}=A$ for $A$ real and so, the second equation has at most two solutions. We claim that we can choose $\rho$ to be positive for these two real solutions and that these solutions are simple. The claim can be verified by the example

$$
\begin{aligned}
& \dot{x}=\varepsilon a x-b y+2 x y^{2} z, \\
& \dot{y}=b x+\varepsilon a y, \\
& \dot{z}=\varepsilon z+a x^{2} y^{2}+\frac{3 z^{4}}{32 a^{3}},
\end{aligned}
$$

that has two periodic solutions $\left(x_{i}(t, \varepsilon), y_{i}(t, \varepsilon), z_{i}(t, \varepsilon)\right)$ for $i=1,2$ such that $\left(x_{1}(0, \varepsilon), y_{1}(0, \varepsilon), z_{1}(0, \varepsilon)\right) \rightarrow(\sqrt{2}, 0,-2 a)$ and $\left(x_{2}(0, \varepsilon), y_{2}(0, \varepsilon), z_{2}(0, \varepsilon)\right) \rightarrow$ $\left(\sqrt{2} \cdot 3^{1 / 6}, 0,-2 a / 3^{1 / 3}\right)$ when $\varepsilon \rightarrow 0$. This completes the proof of Theorem 3 .

\section{Proof of Theorem 4}

Doing the cylindrical change of variables given in (4) in the region $r>0$, system (1) becomes system (5) where now the sum runs over $i_{1}+\cdots+i_{n}=5$. Working as in the proofs of Theorems 2 and 3 system (5) in a neighborhood of $\left(r, z_{3}, \ldots, z_{n}\right)=(0,0, \ldots, 0)$ with $r>0$ becomes system (6) where again the sum runs over $i_{1}+\cdots+i_{n}=5$. Note that this system is periodic or period $2 \pi$ in $\theta$.

To apply the averaging theory we rescale the variables, setting

$$
\left(r, z_{3}, \ldots, z_{n}\right)=\left(\varepsilon^{1 / 4} \rho, \varepsilon^{1 / 4} \eta_{3}, \ldots, \varepsilon^{1 / 4} \eta_{n}\right) .
$$


Then system (1) becomes system (8) with $i_{1}+\cdots+i_{n}=5$. Note that system (8) has the form (2) of the averaging theory. Again working as in the proofs of Theorems 2 and 3 we obtain

$$
\begin{aligned}
f_{1}^{0}= & \frac{\rho}{16 b}\left(16 a+\left(a_{14 \mathbf{0}_{n-2}}+a_{32 \mathbf{0}_{n-2}}+a_{50 \mathbf{0}_{n-2}}+b_{41 \mathbf{0}_{n-2}}+b_{23 \mathbf{0}_{n-2}}+b_{05 \mathbf{0}_{n-2}}\right) \rho^{4}\right. \\
& +\sum_{j=3}^{k} 2\left(a_{12 e_{i j}}+b_{21 e_{i j}}+3\left(a_{30 e_{i j}}+b_{03 e_{i j}}\right)\right) \rho^{2} \eta_{i} \eta_{j} \\
& \left.+8 \sum_{3 \leq i \leq j \leq l \leq u \leq n}\left(a_{10 e_{i j l u}}+b_{01 e_{i j l u}}\right) \eta_{i} \eta_{j} \eta_{l} \eta_{u}\right) \\
f_{k}^{0}= & \frac{1}{8 b}\left(8 c_{k} \eta_{k}+\sum_{j=3}^{n}\left(3 c_{04 e_{j}}^{(k)}+3 c_{40 e_{j}}^{(k)}+c_{22 e_{j}}^{(k)}\right) \rho^{4} \eta_{j}\right. \\
& +4 \sum_{3 \leq i \leq j \leq l \leq n}\left(c_{02 e_{i j l}}^{(k)}+c_{20 e_{i j l}}^{(k)}\right) \rho^{2} \eta_{i} \eta_{j} \eta_{l} \\
& \left.+8 \sum_{3 \leq i \leq j \leq l \leq u \leq v \leq n} c_{00 e_{i j l u v}}^{(k)} \eta_{i} \eta_{j} \eta_{l} \eta_{u} \eta_{v}\right)
\end{aligned}
$$

for $k=3, \ldots, n$, where $e_{i j l u v} \in \mathbb{Z}_{+}^{n-2}$ has the sum of the $i$-th, $j$-th, $l$-th, $u$-th and $v$-th entries equal to 5 and the other entries equal to zero (eventually these entries can coincide).

We need to compute the zeroes of the algebraic equations

$$
\begin{aligned}
& 16 a+\left(a_{14 \mathbf{0}_{n-2}}+a_{32 \mathbf{0}_{n-2}}+a_{50 \mathbf{0}_{n-2}}+b_{41 \mathbf{0}_{n-2}}+b_{23 \mathbf{0}_{n-2}}+b_{05 \mathbf{0}_{n-2}}\right) \rho^{4} \\
& +\sum_{j=3}^{k} 2\left(a_{12 e_{i j}}+b_{21 e_{i j}}+3\left(a_{30 e_{i j}}+b_{03 e_{i j}}\right)\right) \rho^{2} \eta_{i} \eta_{j} \\
& +8 \sum_{3 \leq i \leq j \leq l \leq u \leq n}\left(a_{10 e_{i j l u}}+b_{01 e_{i j l u} l u}\right) \eta_{i} \eta_{j} \eta_{l} \eta_{u}=0, \\
& 8 c_{k} \eta_{k}+\sum_{j=3}^{n}\left(3 c_{04 e_{j}}^{(k)}+3 c_{40 e_{j}}^{(k)}+c_{22 e_{j}}^{(k)}\right) \rho^{4} \eta_{j}+4 \sum_{3 \leq i \leq j \leq l \leq n}\left(c_{02 e_{i j l}}^{(k)}+c_{20 e_{i j l}}^{(k)}\right) \rho^{2} \eta_{i} \eta_{j} \eta_{l} \\
& +8 \sum_{3 \leq i \leq j \leq l \leq u \leq v \leq n} c_{00 e_{i j l u v}}^{(k)} \eta_{i} \eta_{j} \eta_{l} \eta_{u} \eta_{v}=0,
\end{aligned}
$$

for $k=3, \ldots, n$.

By Bezout Theorem, the maximum number of solution that we can have is $4 \cdot 5^{n-2}$. As in the proof of Theorem 3 this upper bound in general is not reached, as we shall see in dimension three. 
We now consider the particular case of $\mathbb{R}^{3}$. In this case we have

$$
\begin{aligned}
f_{1}^{0} & =\frac{\rho}{16 b}\left(16 a+A \rho^{4}+B \rho^{2} \eta_{3}^{2}+C \eta_{3}^{4}\right), \\
f_{2}^{0} & =\frac{\eta_{3}}{8 b}\left(8 c_{3}+D \rho^{4}+F \rho^{2} \eta_{3}^{2}+G \eta_{3}^{4}\right),
\end{aligned}
$$

where

$$
\begin{aligned}
& A=a_{140}+a_{320}+5 a_{500}+b_{410}+b_{230}+5 b_{050}, \\
& B=2\left(a_{122}+b_{212}+3\left(a_{302}+b_{032}\right)\right), \\
& C=8\left(a_{104}+b_{014}\right), \\
& D=3\left(c_{041}+c_{401}\right)+c_{221}, \\
& F=4\left(c_{023}+c_{203}\right), \\
& G=8 c_{005} .
\end{aligned}
$$

We need to compute the zeroes of the system

$$
\begin{aligned}
& 16 a+A \rho^{4}+B \rho^{2} \eta_{3}^{2}+C \eta_{3}^{4}=0, \\
& \eta_{3}\left(8 c_{3}+D \rho^{4}+F \rho^{2} \eta_{3}^{2}+G \eta_{3}^{4}\right)=0 .
\end{aligned}
$$

If $\eta_{3}=0$ then $f_{1}^{0}=0$ yields a unique real positive solution $\rho$ that we write as $\left(\rho_{0}, 0\right)$. Now consider the case $\eta_{3} \neq 0$. We define

$$
\begin{aligned}
& W_{1}=2 \sqrt{\frac{\alpha-\beta}{\delta}}, \\
& R_{1}=\frac{W_{1}\left(-D B^{2}+A F B+2 a D F B-2 a A F^{2}+\beta\right)}{2(2 a D-A)(B D-A F)}, \\
& W_{3}=2 \sqrt{\frac{\alpha+\beta}{\delta}}, \\
& R_{3}=\frac{W_{3}\left(-D B^{2}+A F B+2 a D F B-2 a A F^{2}-\beta\right)}{2(2 a D-A)(B D-A F)},
\end{aligned}
$$

where

$$
\begin{aligned}
& \alpha=2 A C D+A B F-B^{2} D-2 A^{2} G-4 C D^{2} a+2 B D F a-2 A F^{2} a+4 A D G a, \\
& \beta=\sqrt{(B D-A F)^{2}\left(B^{2}-4 B F a-4 A(C-2 G a)+4 a\left(2 C D+F^{2} a-4 D G a\right)\right)}, \\
& \delta=C^{2} D^{2}+G\left(B^{2} D-A B F+A^{2} G\right)+C\left(-B D F+A\left(F^{2}-2 D G\right)\right) .
\end{aligned}
$$

Setting $\left(f_{1}^{0}, f_{2}^{0}\right)=0$ in $R=\rho^{2}$ and $W=\eta_{3}^{2}$ we get four solutions $\left(R_{1}, W_{1}\right),\left(-R_{1},-W_{1}\right),\left(R_{3}, W_{3}\right),\left(-R_{3},-W_{3}\right)$. Since $R=\rho^{2}$ must be positive we get that only two of them are possible. Then we can have five simple solutions $\left(\rho_{0}, 0\right),\left(\sqrt{R_{1}}, \pm \sqrt{W_{1}}\right)$ and $\left(\sqrt{R_{3}}, \pm \sqrt{W_{3}}\right)$ as the following 
example illustrates. System

$$
\begin{aligned}
& \dot{x}=-\frac{\varepsilon}{35} x-b y+\frac{16}{35} x y^{4}+\frac{34}{35} x y^{2} z^{2}, \\
& \dot{y}=b x-\frac{\varepsilon}{35} y, \\
& \dot{z}=\varepsilon z+\frac{y^{4} z}{4}
\end{aligned}
$$

has four periodic solutions $\left(x_{i}(t, \varepsilon), y_{i}(t, \varepsilon), z_{i}(t, \varepsilon)\right.$ for $i=0, \ldots, 4$ such that

$$
\begin{aligned}
& \left(x_{0}(0, \varepsilon), y_{0}(0, \varepsilon), z_{0}(0, \varepsilon)\right) \rightarrow(1,0,0), \\
& \left(x_{1}(0, \varepsilon), y_{1}(0, \varepsilon), z_{1}(0, \varepsilon)\right) \rightarrow\left(\sqrt{2} / 3^{1 / 4}, 0, \sqrt{2} / 3^{1 / 4}\right), \\
& \left(x_{2}(0, \varepsilon), y_{2}(0, \varepsilon), z_{2}(0, \varepsilon)\right) \rightarrow\left(\sqrt{2} / 3^{1 / 4}, 0,-\sqrt{2} / 3^{1 / 4}\right), \\
& \left(x_{3}(0, \varepsilon), y_{3}(0, \varepsilon), z_{3}(0, \varepsilon)\right) \rightarrow(\sqrt{6}, 0, \sqrt{2}),
\end{aligned}
$$

and

$$
\left(x_{4}(0, \varepsilon), y_{4}(0, \varepsilon), z_{4}(0, \varepsilon)\right) \rightarrow(\sqrt{6}, 0,-\sqrt{2}),
$$

when $\varepsilon \rightarrow 0$. This completes the proof of Theorem 4 .

\section{ACKNOWLEDGEMENTS}

The first and third author are partially supported by FCT/Portugal through UID/MAT/04459/2013. The second author is partially supported by a FEDER-MINECO grant MTM2016-77278-P, a MINECO grant MTM201340998-P, and an AGAUR grant 2014SGR-568.

\section{REFERENCES}

[1] A. Buica And J. Llibre, Averaging methods for finding periodic orbits via Brouwer degree, Bull. Sci. Math. 128 (2004), 7-22.

[2] A.R. Champneys And V. KiRK, The entwined wiggling of homoclinic curves emerging from saddle-node/Hopf instabilities. Phys. D 195 (2004), 77-105.

[3] J. Guckenheimer, On a codimension two bifurcation, Lecture Notes in Math. 898 (1980), 99-142.

[4] J. Guckenheimer And P. Holmes, Nonlinear Oscillations, Dynamical Systems and Bifurcations of Vector Fields, Springer, 1983.

[5] Y.A. Kuznetsov, Elements of Applied Bifurcation Theory, Springer-Verlag, 3rd edition, 2004.

[6] J. Llibre And C. VAlls, Hopf bifurcation for some analytic differential systems in $R^{3}$ via averaging theory, Discrete, Continuous Dynamical Systems, Series A 30 (2011), 779-790.

[7] J. Llibre AND X. Zhang, Hopf bifurcation in higher dimensional differential systems via the averaging method, Pacific J.of Math. 240 (2009), 321-341.

[8] N. G. Lloyd, Degree Theory, Cambridge University Press, 1978.

[9] J.E. Marsden And M. MCCracken, The Hopf bifurcation and its applications, Applied Math. Sciences 19, Springer, New York, 1976. 
[10] J.A. Sanders, F. Verhulst And J. Murdock, Averaging methods in nonlinear dynamical systems, Second edition, Applied Mathematical Sciences 59, Springer, New York, 2007.

[11] J. Scheurle And J. Marsden, Bifurcation to quasi-periodic tori in the interaction of steady state and Hopf bifurcations, SIAM. J. Math. Anal. 15 (1984), 1055-1074.

[12] I.R. Shafarevich, Basic Algebraic Geometry, Springer, 1974.

[13] Z.F Zhang, T.R. Ding, W.Z. Huang And Z.X. Dong, Qualitative theory of differential equations, Translations of Math. Monogr. 101, Amer. Math. Soc. Providence, R.I., 1992.

${ }^{1}$ Departamento de Matemática, Instituto Superior Técnico, Universidade Técnica de Lisboa, Av. Rovisco Pais 1049-001, Lisboa, Portugal

E-mail address: barreira@math.ist.utl.pt, cvalls@math.ist.utl.pt

2 Departament de Matemàtiques, Universitat Autònoma de Barcelona, 08193 Bellaterra, Barcelona, Catalonia, Spain

E-mail address: jllibre@mat.uab.cat 